\title{
A non-invasive navigation system for retargeting gastroscopic lesions
}

\author{
Jiquan Liu ${ }^{\mathrm{a}, \mathrm{b}, *}$, Bin Wang ${ }^{\mathrm{a}, \mathrm{b}}$, Weiling $\mathrm{Hu}^{\mathrm{c}}$, Yun Zong ${ }^{\mathrm{a}, \mathrm{b}}$, Jianmin $\mathrm{Si}^{\mathrm{c}}$ and Huilong Duan ${ }^{\mathrm{a}, \mathrm{b}}$ \\ ${ }^{a}$ College of Biomedical Engineering \& Instrument Science, Zhejiang University, Hangzhou 310027, \\ China \\ ${ }^{b}$ Key Laboratory for Biomedical Engineering, Ministry of Education, Zhejiang University, Hangzhou \\ 310027, China \\ ${ }^{c}$ Department of Gastroenterology, Sir Run Run Shaw Hospital, Zhejiang University, Hangzhou 310016, \\ China
}

\begin{abstract}
Biopsy is a traditional endoscopic surveillance of premalignant gastric lesions, and endoscopic tattooing is used for marking the biopsy's location. However, the tattooing has several disadvantages. For example, the procedure is an invasive operation and may not be durable due to the diffusion. Moreover, it is procedurally cumbersome with an associated risk of technical failure. In this study, a computer aided endoscopic navigation system (CAEN system) was developed for a non-invasive biopsy procedure. The CAEN system consists of a new, designed six degree of freedom (6-DOF) tracking endoscope device and a computer simulated work station. During the procedure, the endoscopist uses the tip of the tracking endoscope to touch the lesion. Then, the lesion's location is recorded in the work station, which then guides the endoscopist in retargeting the lesions in the follow-ups. The clinical experimental results demonstrate that the accuracy at the angularis is $5.2 \pm 2.8 \mathrm{~mm}$, at the antral lesser curvature is $7.2 \pm 2.0 \mathrm{~mm}$, at the antral greater curvature is $6.3 \pm 3.1 \mathrm{~mm}$, at the antral posterior wall is $8.2 \pm 1.6 \mathrm{~mm}$, and at the antral anterior wall is $7.9 \pm 1.3 \mathrm{~mm}$. The mean accuracy is $7.5 \mathrm{~mm}$, and the P-value is 0.023 , which is likely suitable for clinical practice. Furthermore, the proposed CAEN system requires less procedural time than the tattooing.
\end{abstract}

Keywords: Endoscopic biopsy marking, non-invasive procedure, 6-DOF endoscope device, hand-eye calibration, computer simulated work station

\section{Introduction}

Gastric cancer is the second most common cause of cancer-related death worldwide [1]. Because gastric cancer is difficult to be diagnosed in its early stage, biopsy becomes critical for confirming gastric cancer. For gastric biopsies, tattooing is the most common biopsy marking and surveillance method. To the authors' knowledge, the average diameter of the tattooing area is almost $1 \mathrm{~cm}$, which indicates that the accuracy of tattooing is around $1 \mathrm{~cm}$. However, tattooing requires injecting India ink into the lesions, which results in some disadvantages: 1 . Over time, the ink may fade, particularly after several months, thus the lesions must be re-marked in the follow-ups; 2 . The ink injection procedure is

\footnotetext{
${ }^{*}$ Corresponding author: Jiquan Liu, College of Biomedical Engineering \& Instrument Science, Zhejiang University, Hangzhou 310027, China. Tel.: 13588899165; Fax:+8657187951960; E-mail: liujq@zju.edu.cn.
} 
cumbersome and has a risk of technical failure; 3. Tattooing cannot navigate the lesion's location in the follow-ups, indicating that it may be necessary to retarget the pathological lesions in the follow-ups depending upon the endoscopist's expertise.

Therefore, some researchers have focused on biopsy surveillance by using computer simulation and optical instruments. For example, B. Allain proposed a biopsy re-localization method based on epipolar geometry and evaluated the results' accuracy by covariance matrix [2], this evaluation indicated that the accuracy depends on the camera's movement, thus limiting the application of this method. D. Sun [3] designed a non-invasive biopsy marking system for a gastroscopy, that is, a computed tomography $(\mathrm{CT})$ virtual 3D model, which facilitates the navigation and biopsy retargeting, but could not accurately determine the stomach's deformations. Additionally, Yang [4] proposed a novel, online deformation method for pathological site retargeting, where the stomach's motion was regarded as a local affine transformation; however, to the authors' knowledge, a local affine assumption could not completely ensure both reliability and consistency. Finally, the probe-based confocal laser endomicroscopy (pCLE) [5] was developed quickly because it is non-invasive and is conducted in real-time; nevertheless, retargeting the previous biopsy site remains challenging.

In this paper, a computer aided endoscopic navigation system (CAEN system) is proposed. By integrating the endoscope with an electromagnetic tracking sensor, the biopsy sites are recorded in a global coordinate instead of a traditional invasive operation. During the gastroscopy and the subsequent follow-ups, all the biopsy sites are marked in the captured gastroscopy video in order to assist the endoscopist in identifying the target sites. Moreover, because of its tiny nature (diameter is $1.3 \mathrm{~mm}$ ), the extra tracking sensor is tolerated well by the patients without increasing operation difficulties for endoscopists. In this study, there were 22 participating volunteers. The experimental results reveal that the CAEN system takes less operation time than the tattooing method. Additionally, the CAEN system's error for the antrum and angularis is less than $1 \mathrm{~cm}$, which is likely suitable for clinical practice since a biopsy forceps' span is nearly $6 \mathrm{~mm}$, and the tattooing area's mean diameter is $1 \mathrm{~cm}$ or more due to diffusion [6]. Moreover, the CANE system is potentially applicable to the retargeting of lesion sites in the bladder, colon, or other cavity organs. In the future, the CAEN system's accuracy will be estimated in a larger number of patients, and the accuracy will be improved for the overall stomach.

\section{Experimental materials and methods}

\subsection{Materials}

The CANE system consists of: an electronic unit device, a sensor probe, a magnetic transmitter, an endoscope (Olympus GIF-QX260), and a CAEN navigation work station. The electronic unit device, sensor probe, and magnetic transmitter (Ascension Technology Corp, Burlington, Vermont, USA) are integrated as a 6-DOF electromagnetic tracking endoscope device (see Figure 1 1-1). The probe is $1.3 \mathrm{~mm}$ in diameter and $6.5 \mathrm{~mm}$ in length, which ensures the operative flexibility, and it is attached to the tip of the endoscope so as to provide real-time position information during the procedure (see Figure 1 1-2). The magnetic transmitter's effective position region is $50 \mathrm{~cm} * 50 \mathrm{~cm} * 50 \mathrm{~cm}$, and it is placed near the patient's abdomen in order to ensure that the probe is within the effective magnetic region during the procedure (see Figure 1 1-3). 

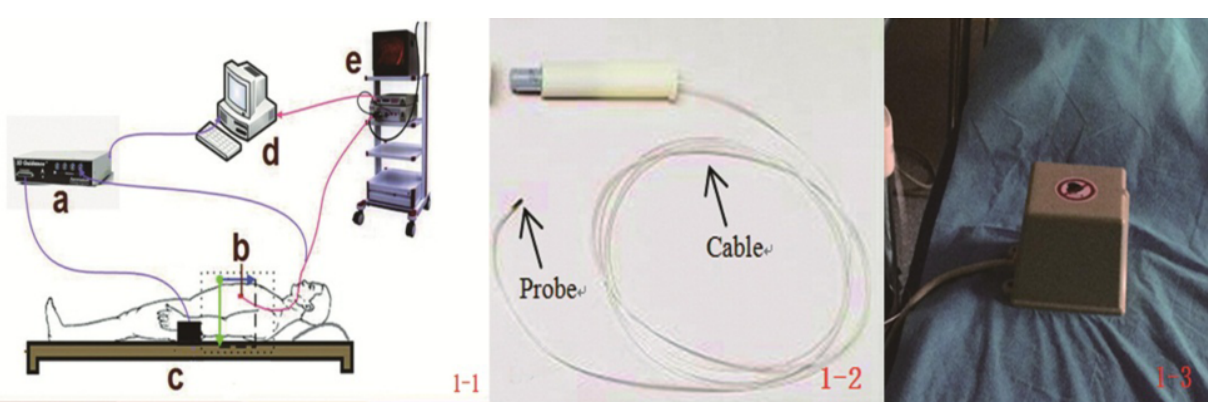

Fig.1. System components of the CAEN system. 1-1 is the workstation, 1-2 is the probe as well as 1-3 is the magnetic transmitter.

\subsection{Hand-eye calibration of the 6-DOF tracking endoscope device}

Before the gastroscopy procedure, the sensor probe is attached to the endoscope's tip. The coordinate system contains the transmitter coordinate system, sensor coordinate system, and endoscopic coordinate system. Due to the inability to annotate reference points on the patient's organ

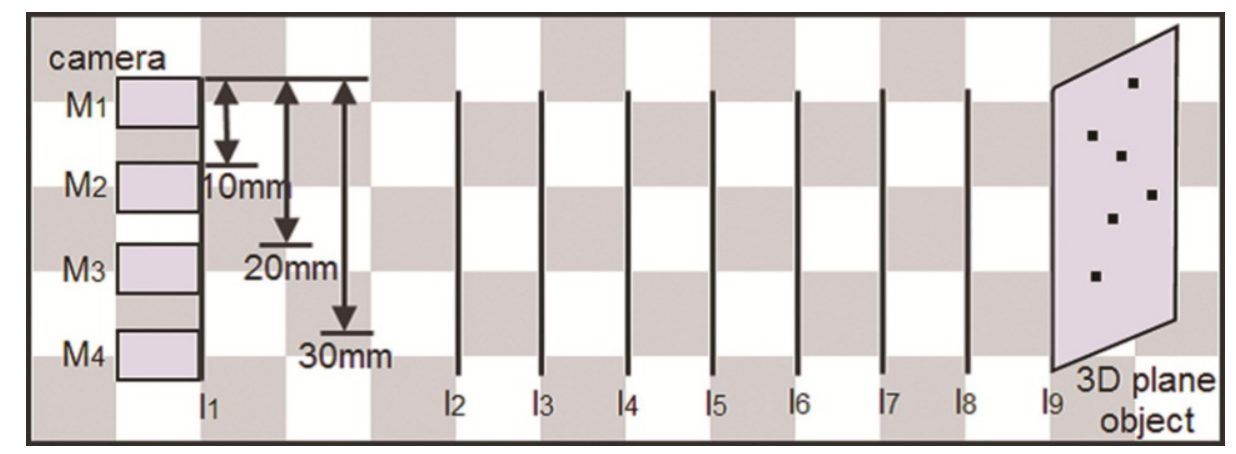

Fig. 2. Hand-eye calibration method for estimating the transform between the sensor and endoscope.

and the immovability of the transmitter's coordinates during the procedure, the transmitter coordinate system is considered to be a global system. Therefore, the endoscope's position and sensor probe information should be estimated in the transmitter coordinate system. Since the transform between the sensor and global coordinates is directly provided by the tracking system, the transform between the sensor and endoscope should be estimated. For robotic research, this transform is considered to be hand-eye calibration [7], and as such, many researchers have presented various schemes to solve this problem $[8,9]$. Unfortunately, it is difficult to apply these schemes to clinical practice since humans cannot process actions as accurately as robots. In this paper, the parallel binocular stereo algorithm is utilized to propose a new method for solving the 6-DOF tracking endoscope's hand-eye problem. The method is illustrated in Figure 2.

In Figure 2, M1 to M4 indicate the endoscope's viewpoints, and 12 to 19 indicate the 3D plane object's positionduring the calibration. Some obvious corners are marked on the plane object as key features. The endoscope and plane object move parallel to the checker-board's edge, which ensures that the endoscope's optical axis remains parallel between different viewpoints as well as vertical to the plane object. During the calibration procedure, the endoscope is located at M1 and acquires images from the 3D plane object at position 12, and then the endoscope moves from M1 to M2, M3, and M4, 
respectively, and acquires images from 12. The key features' coordinates are estimated in the endoscope camera's coordinates as:

$$
Z_{i, M}=\frac{2 f_{x} d}{x_{i, M}-x_{i, N}}, X_{i, M}=\frac{Z_{i, M}}{f_{x}}\left(x_{i, M}-c_{x}\right), Y_{i, M}=\frac{Z_{i, M}}{f_{y}}\left(y_{i, M}-c_{y}\right)
$$

In Eq. (1), indicate the endoscope camera's intrinsic parameters, which are estimated by Zhang's method [10],are the pixel coordinates of the key feature i with the endoscope located at $\mathrm{M}$; are the endoscope camera's coordinates of the key feature i with the endoscope located at M; and indicate the disparity. The global coordinates of the marked points are measured by directly touching them with the sensor. As a result, the global-to-camera coordinate transform can be estimated with the corresponding camera's coordinates and the key features' global coordinates.

The acquirements are repeated with the endoscope located at the 12,13..19 locations. For each location, a set of global-to-camera transforms are calculated, and afterwards, RANdom SAmple Consensus (RANSAC) is used to select the most reliable transform.

\subsection{Computer simulated work station}

The work station's work flow is described in Figure 3. The input for the CAEN system work station is a series of endoscopic image sequences. Prior to examination, the work station requires that an endoscopist should touch five reference points with the tip of the 6-DOF endoscope device (the five points being the cardia, junction of the anterior body wall and angularis, junction of the posterior body wall and angularis, middle of the antrum, and pylorus). The five reference points are used for global coordinate registration for different examination procedures. In this paper, a seven-parameter Burse-Wolf formula is used to estimate the 3D registration model. During the first examination, the biopsy sites are touched by the tip of the 6-DOF endoscope device, and the global coordinates are then recorded by the work station. During the follow-up examinations, the global coordinate's registration is estimated. Afterwards, the recorded biopsy sites' positions are transformed and then marked in real endoscopic videos in order to guide the endoscopist in retargeting the biopsy sites. To visualize the navigation, a virtual 3D stomach model is constructed after the first examination by the simultaneous localization and mapping (SLAM) method [11]. During the follow-up examinations, the biopsy sites and the endoscope tip's position are visualized in order to assist the endoscopist in finding the biopsy sites (see Figure 4). 


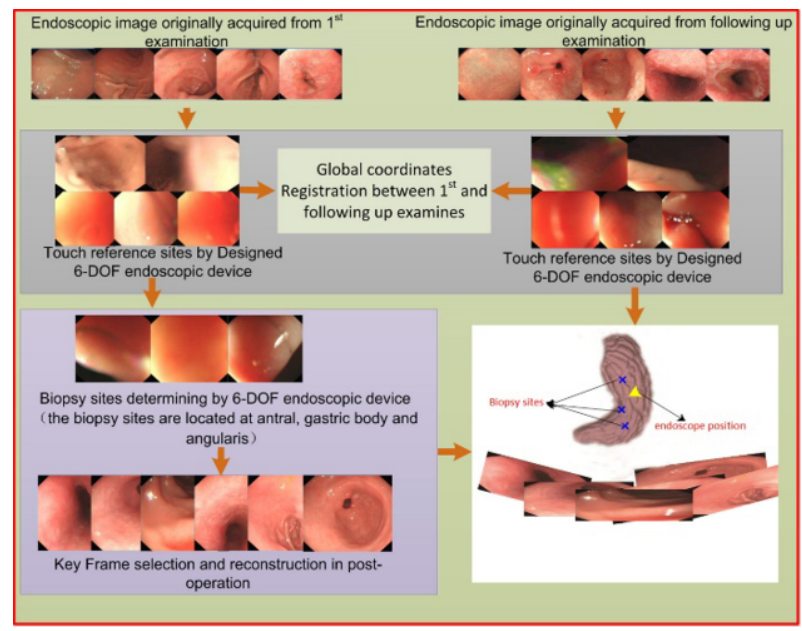

Fig. 3. CAEN system's work station.

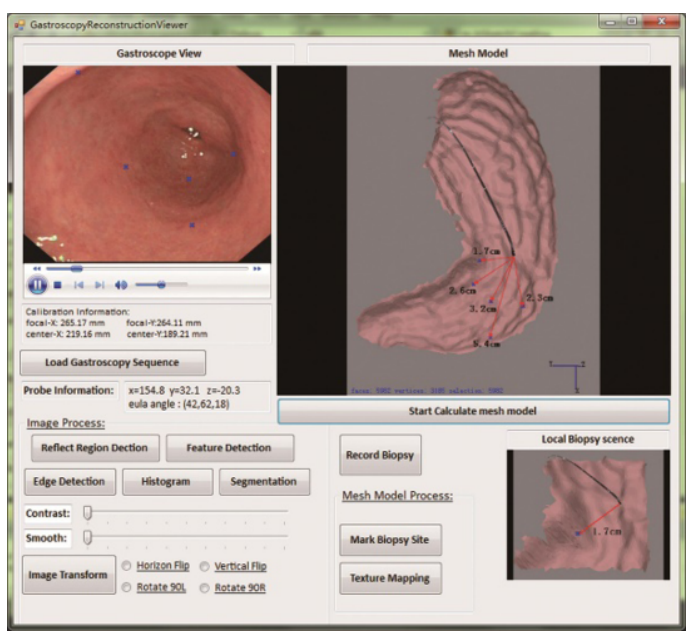

Fig. 4. UI of CAEN system for follow-up examination.

In Figure 4, the image in the top-left shows the real gastroscopy sequence, with blue marks indicating the guided biopsy sites. The image in the top-right shows the virtual global mesh model of the stomach. The biopsy sites, the endoscope's position and the distance between them are marked on the model in real-time in order to assist the endoscopist in retargeting the biopsy sites. The local biopsy view (the bottom-right) shows the distance between the endoscope and the nearest biopsy site.

\section{Results}

To validate the CAEN system's accuracy, the system was installed in the Sir Run Run Shaw Hospital in Zhejiang province, China. The endoscope model was GIF-QX 260 EGD from Olympus Corp., Japan. For the study, 22 patients with histories of moderate to severe gastric intestinal metaplasia were enrolled. In consideration of their safety, none of the patients had pacemakers or other medical devices that were sensitive to magnets. All of the patients provided a written informed consent for the evaluation and for follow-up medical records. The gastroscopy examination was performed by an endoscopist according to the conventional clinical protocol. All of the patients enrolled with ease and were without any adverse events (see Table 1).

Table 1

Clinical characteristics

\begin{tabular}{|c|c|}
\hline Variables & Number \\
\hline Median age, year(range) & $58.3(40-77)$ \\
\hline Sex(female/male) & $6 / 16$ \\
\hline Smoking & 11 \\
\hline Alcohol & 14 \\
\hline EGD pathology(Moderate IM/ Severe IM) & $3 / 19$ \\
\hline
\end{tabular}

EGD: esophagogastric dissociation

IM: intestinal metaplasia 
Table 2

Accuracy of the CAEN system

\begin{tabular}{||llc||}
\hline Biopsy Location & Accuracies:(mean \pm SD) & $\begin{array}{c}\text { Marking time (mean } \pm \text { SD)s } \\
\text { (Tattooing/CAEN) }\end{array}$ \\
Angularis & $\mathrm{mm}$ & $12 \pm 4.1 / 3 \pm 1.8$ \\
Antral lesser curvature & $7.2 \pm 2.8$ & $12 \pm 4.4 / 2 \pm 0.8$ \\
Antral greater curvature & $6.3 \pm 3.1$ & $11 \pm 3.6 / 2 \pm 0.7$ \\
Antral Posterior wall & $8.2 \pm 1.6$ & $12 \pm 5.5 / 2 \pm 0.7$ \\
Antral Anterior wall & $7.9 \pm 1.3$ & $13 \pm 5.2 / 2 \pm 0.7$ \\
\hline
\end{tabular}

Prior to examination, the sensor probe was attached to the endoscope by surgical tape, and the probe was sterilized the same as the endoscope. Before the procedure, the hand-eye calibration was estimated. After the stomach was intubated, the reference points were first touched by the 6-DOF endoscopy device; afterwards, the five biopsy sites were selected, that is, the points being the lesser curvature of the antrum, greater curvature of the antrum, angularis, anterior wall, and posterior wall of the antrum. The selected biopsy sites were then marked by tattooing and the 6-DOF endoscopy device, respectively.

Three months after the first examination, a follow-up endoscopy was performed with the CAEN system, and the global coordinates' registration was estimated between the first and follow-up examinations. Subsequently, the CAEN system navigated the endoscopist to retarget the biopsy site. The distance between the targeted points by the CAEN system and the center of the tattooing area was designated as the accuracy of the CAEN system. Moreover, the CAEN system's touching time and tattooing time were recorded. The mean accuracy of the CAEN system was $7.5 \mathrm{~mm}$, and the operation time was much shorter than the tattooing time (see Table 2).

The distance between the tattooing center point and the CAEN target point was directly measured as pixel unit in the image. Additionally, the diameter of the biopsy forceps was considered to be $6 \mathrm{~mm}$, which provided a gold standard for converting the accuracy from pixels to millimeters.

\section{Discussion}

In this paper, a novel system was studied and developed for a non-invasive biopsy navigation. The clinical study revealed that the mean accuracy was likely suitable for clinical practice. Furthermore, the touching time was much shorter than that for the injection, which indicates that the CAEN system has the potential to shorten the operation time, reduce patients' trauma, and improve recovery time. In this study, 22 patients and a skilled endoscopist were enrolled, and further studies will be conducted in order to validate the accuracy in a larger number of patients and endoscopists.

To visualize the navigation scene, a 3D stomach model was constructed based on SLAM. For the deformation, specular reflection, and partial occlusion of the stomach, the CAEN system cannot currently construct the 3D model without human intervention. However, in the authors' opinion, this does not influence the navigation's accuracy in the follow-ups. In the future, the primary focus will be on optimizing the technical scheme so as to improve the accuracy and realize non-invasive biopsy navigation in the overall stomach (e.g., the gastric body). This future work can be divided into two parts:

1. Hand-eye calibration's integration. Currently, the sensor probe is attached to the endoscope by surgical tape, which indicates that the hand-eye calibration should be performed before every 
gastroscopy procedure, thus increasing the probability of error. In the future, the sensor probe will be incorporated into the endoscope as a professional endoscope device.

2. Global coordinate registration optimization. The transform between the first examination's global coordinates and the follow-up's global coordinates was estimated by touching the five reference points during the procedure, and the accuracy of the touching depended upon the endoscopist's expertise. Furthermore, the current selected reference points cannot accurately represent the gastric body's deformation, and for this reason, the CAEN system cannot be applied to the gastric body. In the future, more reference points will be exploited based on advanced image feature detectors so as to make the registration more accurate and convenient.

\section{Acknowledgement}

The authors thank Dr. Jianmin Si and Dr. Weiling Hu from the Sir Run Run Shaw Hospital, Hangzhou, for their help in improving the algorithm. The project was supported by the National Key Technology Support Program (No. 2012AA02A606) and the Fundamental Research Funds for the Central Universities (No. 2013QNA5018).

\section{References}

[1] A. Jemal, F. Bray, M.M. Center, J. Ferlay, E. Ward and D. Forman, Global cancer statistics, CA: A Cancer Journal for Clinicians 61 (2011), 69-90.

[2] B. Allain, M. Hu, L.B. Lovat, R.J. Cook, T. Vercauteren, S. Ourselin and D. J Hawkes, Re-localisation of a biopsy site in endoscopic images and characterisation of its uncertainty, Medical Image Analysis 16 (2012), 482-496.

[3] D. Sun, W. Hu, W. Wu, J. Liu, H. Duan and J. Si, Design of the image-Guided biopsy marking system for gastroscopy, Journal of Medical Systems 36 (2012), 1-12.

[4] M. Ye, S. Giannarou, N. Patel, J. Teare and G.-Z. Yang, Pathological site retargeting under tissue deformation using geometrical association and tracking, Medical Image Computing and Computer-Assisted Intervention-MICCAI 2013 8150 (2013), 67-74.

[5] U. Ladabaum, A. Fioritto, A. Mitani, M. Desai, J.P. Kim, D.K. Rex, T. Imperiale and N Gunaratnam, Real-time optical biopsy of colon polyps with narrow band imaging in community practice does not yet meet key thresholds for clinical decisions, Gastroenterology 144 (2013), 81-91.

[6] R. Krishnappa, M. Horakerappa, K. Ali and M. Gouri, A study on histopathological spectrum of upper gastrointestinal tract endoscopic biopsies, International Journal of Medical Research \& Health Sciences 2 (2013), 418-424.

[7] R. Horaud and F. Dornaika, Hand-eye calibration, The International Journal of Robotics Research 14 (1995), $195-210$.

[8] F. Chun, W. Hong-Tao, Q. Bing and H. Peng, Combining camera calibration with hand-eye calibration and using in monocular vision, 2010 International Conference on Computer, Mechatronics, Control and Electronic Engineering (CMCE) 6 (2010), 21-24.

[9] J. Heller, M. Havlena, A. Sugimoto and T. Pajdla, Structure-from-motion based hand-eye calibration using L $\infty$ minimization, 2011 IEEE Conference on Computer Vision and Pattern Recognition (CVPR) 2011, 3497-3503.

[10] Z.Y. Zhang, Flexible camera calibration by viewing a plane from unknown orientations, The Proceedings of the Seventh IEEE International Conference on Computer Vision 1 (1999), 666-673.

[11] S. Thrun and J.J. Leonard, Simultaneous localization and mapping, Springer Handbook of Robotics, 2008, pp. 871-889. 